\title{
Breath Amplitude Modulation of Heart Rate Variability in Normal Full Term Neonates
}

\author{
FRANCINE D. DYKES, PETER A. AHMANN, KARIN BALDZER, TIMOTHY A. CARRIGAN, \\ RICHARD KITNEY, ${ }^{1}$ AND DON P. GIDDENS ${ }^{2}$ \\ Department of Pediatrics, Divisions of Neonatal-Perinatal Medicine and Pediatric Neurology, Emory University \\ School of Medicine, Atlanta 30322
}

\begin{abstract}
The relationship between heart rate variability and respiration patterns was investigated using spectral analysis techniques in nine full-term infants whose ages ranged from 39-75 h. All the infants were studied during sleep, although no attempt was made to classify rapid eye movement or nonrapid eye movement states prospectively. The data obtained were examined to determine which aspects of neonatal breathing patterns are correlated with heart rate variability. Three spectral regions of heart rate variability could be identified: a very low frequency region below $0.02 \mathrm{~Hz}$; a low frequency region from $0.02-0.20 \mathrm{~Hz}$; and a high frequency region above $0.20 \mathrm{~Hz}$. The dominant heart rate variability activity in these neonates was seen in the very low and low frequency regions, with little activity in the high frequency regions. In contrast to older infants and adults, respiration and heart rate variability were not strongly related through a high frequency region respiratory sinus arrhythmia but rather through a breath amplitude sinus arrhythmia which occurs in the low frequency region of the spectrum. The prominent very low frequency activity and the low frequency activity ascribed to breath amplitude modulation may result from autonomic nervous system mediation of chemoregulation. (Pediatr Res 20: 301-308, 1986)
\end{abstract}

\section{Abbreviations}

HRV, heart rate variability

RSA, respiratory sinus arrhythmia

REM, rapid eye movement

AR, autoregressive

VLF, very low frequency

LF, low frequency

HF, high frequency

BA, breath amplitude

BASA, breath amplitude sinus arrhythmia

HR, heart rate

CCF, cross-correlation function

HRV is affected by numerous factors including blood pressure, temperature, respiration, biochemical influence of acid-base balance, state of oxygenation, ventilation, physical movement, and

Received April 9, 1985; accepted November 26, 1985.

Correspondence and reprints Francine D. Dykes, M.D., Emory University Scool of Medicine, Department of Pediatrics, Division of Neurology, 2040 Ridgewood Drive, N.E., Atlanta, GA 30322.

This work was supported by a grant-in-aid from the Georgia Affiliate of the American Heart Association and from a focus giving grant from the Johnson \& Johnson Corporation.

${ }^{1}$ Present address Imperial College of Science and Technology, London, England.

${ }^{2}$ Present address The Georgia Institute of Technology, Atlanta, GA. psychological parameters. Thus, HRV arises from neural and extraneural origins, making it complex to employ HRV patterns as a measure of autonomic function. Despite this complexity, definite relationships between HRV and certain other physiological variables are now well established for normal adults. For example, RSA has been documented for many years (1). Furthermore, studies using time series analysis and control theory (e.g. 2-5) have shown that the frequency content of the HR waveform offers considerably more information on cardiovascular regulation than simpler measures such as short-term and long-term variability parameters (6). For example, several investigators $(7,8)$ have identified three regions of activity in the spontaneous HRV spectrum of healthy adults: a LF region around $0.05 \mathrm{~Hz}$ due to thermoregulation; a component near 0.1 $\mathrm{Hz}$ arising from baroreceptor activity; and a component at the breathing frequency (i.e. respiratory sinus arrhythmia), typically around $0.25 \mathrm{~Hz}$ ( 15 breaths/min).

Knowledge of HRV in neonates is less extensive. Porges (9) has discussed the diagnostic potential of HRV, pointing out that it may be possible to utilize more quantitative techniques such as spectral analysis to obtain a measure of autonomic function. For example, Porges (9) has employed power spectral density estimates of heart period activity associated with the respiratory frequency band as a measure of RSA amplitude and used this to estimate vagal tone. Nugent and Finley (10) have shown correspondence between the frequency of periodic breathing and HRV in neonates, while Kitney (11) reported a common frequency in the spectra of $\mathrm{HR}$, respiratory amplitude, and breath duration records of a 6-wk-old infant. Haddad et al. (12) studied RSA in 4-wk-old puppies and adult dogs and concluded that the physiological mechanisms involved mature postnatally. They noted that LF oscillations, unrelated to the breathing rate, characterize HRV in early life but are insignificant in adult dogs. Experience in a lamb model (13) implies that the sympathetic and parasympathetic nervous systems mature at different rates with the sympathetic nervous system dominant in fetal life. The two systems are nearly balanced at term birth with the parasympathetic nervous system becoming increasingly dominant after birth. Gordon et al. (14) report that the sympathetic nervous system appears responsive only at frequencies below approximately $0.1 \mathrm{~Hz}$, whereas the parasympathetic nervous system is additionally capable of mediation at higher frequencies.

Neither HRV nor respiration patterns have been described adequately in newborns using frequency analysis methods, nor has there been a study of the relationships between these patterns. Work in animal models (12-14) suggests that the spectral description is related to neurological development and may offer the possibility of determining certain aspects of autonomic function. Consequently, the long range objective of this investigation is to determine normative data for neonatal $\mathrm{HRV}$ and respiration patterns, to relate these patterns to physiological control mechanisms, and to determine the usefulness of spectral analysis as 
an aid to perinatal care. Toward this objective, the specific purpose of this study was to investigate relationships between HR and respiration patterns in the normal term infant during the first few days of postnatal life and to identify frequency components which are common to both.

\section{METHODS}

Study infants were nine full term infants $(38-42 \mathrm{wk}$ gestational age) selected over a 2-month interval who had Apgar scores greater than 7 at 1 and 5 min and had no congenital anomalies. Any maternal drug exposure or evidence of acute or chronic fetal distress excluded an infant from study. Informed consent was obtained from the mothers to record the ECG and respirations of these infants according to a protocol approved by the Emory University Human Investigation Committee. The studies were performed between 39 and $75 \mathrm{~h}$ of age in the term nurseries at Grady Memorial Hospital in Atlanta, GA. The infants remained in their own beds with monitoring electrodes attached in the standard three lead manner. No prospective attempt was made to classify REM or non-REM sleep. The ECG and respirations were detected with a Hewlett Packard $78201 \mathrm{~B}$ neonatal monitor. The respiratory waveform is a measure of the excursion of the chest during the respiratory cycle and is derived from the changes in electrical impedance of the chest wall between the ECG electrodes as the infant breathes. The analog signals of ECG and respirations were then recorded on a 4 channel RACAL Store-4 FM instrumentation tape recorder at a tape speed of 15/16 IPS.

The ECG data were digitized, the peaks of the QRS complex detected and the reciprocal of the $R R$ interval used as the "instantaneous" value of HR. When examining HR and respiration together, both channels of data were digitized simultaneously. The HR and respiration data were spectrally analyzed to determine their distribution of power as a function of frequency (power spectral density) and to ascertain whether common frequency patterns occur in these signals.

It often proved useful to examine relationships between heart rate and respiration patterns in the time domain as well as the frequency domain. For this purpose, CCFs were computed (15). Frequencies of oscillation which are common to two signals can be identified readily in the CCF. For example, when two curves which each oscillate at a given frequency are cross-correlated, the resulting $\mathrm{CCF}$ is oscillatory at that common frequency.

Additional details of the methods of acquiring and analyzing the data are provided in the Appendix.

\section{RESULTS}

$H R$ and its spectrum. The power spectral density functions of HR waveforms were studied in nine healthy, full-term neonates. Details of the spectra showed considerable variation both within a given subject and across the population. However, despite this variability certain features emerged as being characteristic of normal HRV in these term infants.

Examination of the data from all nine infants showed that globally HRV could be divided into three spectral regions: (i) a VLF region below ca $0.02 \mathrm{~Hz}$; (ii) a $\mathrm{LF}$ region from ca 0.02 $0.20 \mathrm{~Hz}$; (iii) a $\mathrm{HF}$ region above ca $0.20 \mathrm{~Hz}$

Table 1 shows the mean HR, SD of HR and power distribution within these three spectral regions for each infant studied. A standard record length of $250 \mathrm{~s}$ was used for each power spectral density determination listed in Table 1 , and in several babies more than one such record was employed to obtain the average power distribution. These records were free of artifact or significant trend and were taken during a time when the respiratory rate was relatively regular. Specific criteria for selecting these records are given in the Appendix.

Figure $1 a$ is an example of HRV data from one infant at age $51 \mathrm{~h}$, recorded for approximately $5 \mathrm{~min}$. Visual inspection of the record reveals that a number of superimposed oscillations of different frequencies are present. First, there are obvious underlying variations at very low frequency as evidenced by the four sections marked $a-d$. These are oscillations of approximately $60-$ s duration $(0.017 \mathrm{~Hz})$. Next, there appears to be notable activity at somewhat higher frequencies, approximately 12- $\mathrm{s}$ duration $(0.083 \mathrm{~Hz})$, as exemplified by the sections labeled $e$ and $f$. Finally, there is a very low amplitude oscillation occurring at frequencies in the region of the respiratory rate which varied from 0.6 to 0.8 $\mathrm{Hz}$ for this infant. In order to define these frequencies more accurately the power spectrum of Figure $1 a$ was calculated and is shown in Figure $1 b$, using a logarithmic scale for the ordinate. Referring to the latter figure, there is a large component $(a)$ between 0.01 and $0.02 \mathrm{~Hz}$, corresponding to the observed VLF behavior. In several of the data records studied this VLF region contains the dominant peak within the HRV spectra. Additionally, Figure $1 b$ exhibits several notable peaks between 0.02 and $0.20 \mathrm{~Hz}$ with a large peak at approximately $0.08 \mathrm{~Hz}$ (b). The power in the frequency region corresponding to the respiratory rate $(0.6-0.8 \mathrm{~Hz}$ or $36-48$ breaths $/ \mathrm{min})$ is very small and would not be observable with a linear scale for power.

Another example of HRV, taken from an infant $39 \mathrm{~h}$ of age, is presented in Figure 2. The same general phenomena are seen, i.e. oscillatory activity falls into approximately three frequency bands. In this case, the high frequency oscillations at the respiratory rate $(0.5-0.8 \mathrm{~Hz}$ or $30-48$ breaths/min) are somewhat larger in amplitude than for the first infant, indicating a greater degree of respiratory sinus arrhythmia. However, this RSA component is quite small relative to the power seen in the lower frequency regions of the spectrum.

$H R$ variability and respiration. Although the $H R V$ spectra consistently contained relatively low power at the respiratory

Table 1. Relative power distribution for three frequency regions in spectral content of HR in nine term neonates

\begin{tabular}{|c|c|c|c|c|c|c|c|}
\hline \multirow[b]{2}{*}{ Infant } & \multirow{2}{*}{$\begin{array}{l}\text { No. of } 250 \mathrm{~s} \\
\text { records }\end{array}$} & \multirow{2}{*}{\multicolumn{2}{|c|}{$\begin{array}{c}\text { Mean HR } \\
\text { bpm (hz) }\end{array}$}} & \multirow[b]{2}{*}{ SD } & \multicolumn{2}{|c|}{$\%$ Total power } & \multirow[b]{2}{*}{$f>0.2$} \\
\hline & & & & & $0<f<0.02$ & $0.02<f<0.2$ & \\
\hline $\mathrm{B}$ & 2 & 123 & $(2.05)$ & \pm 0.10 & 38.6 & 56.2 & 5.2 \\
\hline $\mathrm{C}$ & 1 & 137 & $(2.28)$ & \pm 0.17 & 39.8 & 53.2 & 7.0 \\
\hline $\mathrm{D}$ & 1 & 127 & $(2.11)$ & \pm 0.11 & 19.5 & 74.4 & 5.8 \\
\hline$E$ & 2 & 141 & $(2.35)$ & \pm 0.12 & 13.8 & 83.5 & 2.7 \\
\hline $\mathrm{H}$ & 3 & 121 & $(2.01)$ & \pm 0.17 & 36.5 & 60.5 & 3.0 \\
\hline I & 2 & 138 & $(2.30)$ & \pm 0.14 & 45.9 & 50.5 & 3.6 \\
\hline Mean & & & & & 34.7 & 60.2 & 5.1 \\
\hline SD & & & & & \pm 16.3 & \pm 15.2 & \pm 2.7 \\
\hline
\end{tabular}



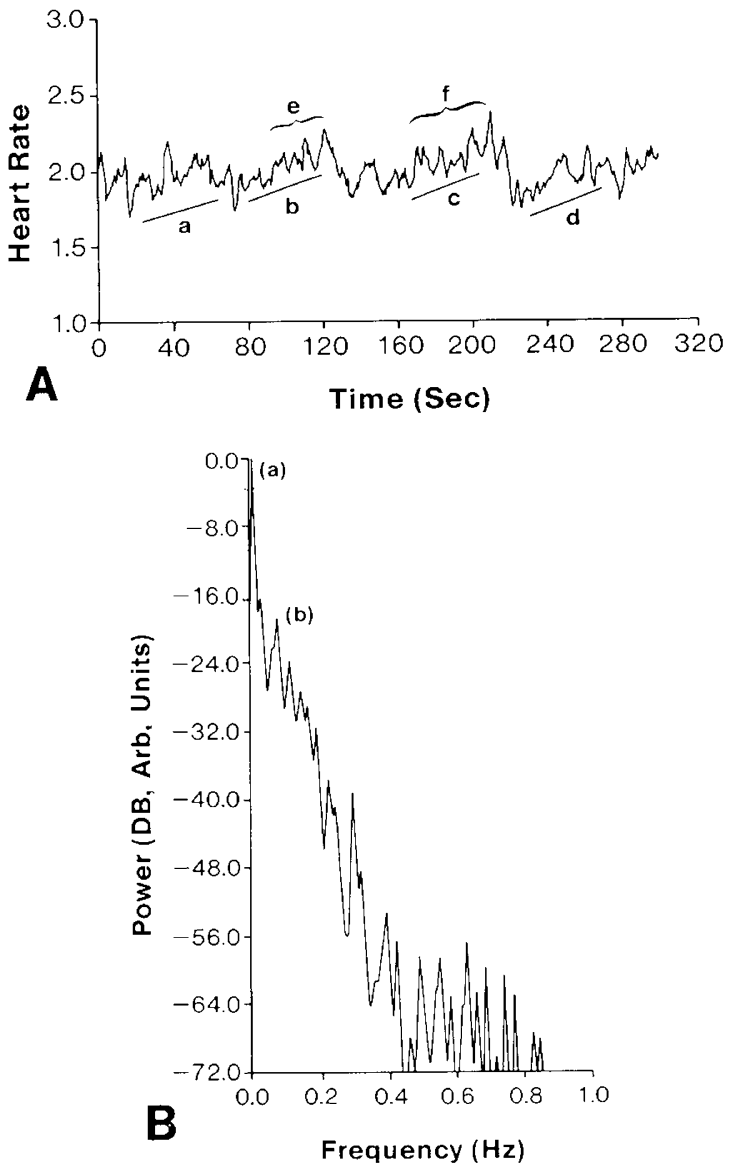

Fig. 1. $a, \mathrm{HR}$ as a function of time for a healthy term infant (infant $\mathrm{F}$, Table 1) at age $51 \mathrm{~h}$. The curve gives the impression of three frequency bands of activity. A very low frequency of HRV can be seen in the regions denoted by $a, b, c$, and $d$, while a higher frequency oscillation is evident as shown by the regions $e$ and $f$. Although not labeled as such, close inspection of the record indicates very small HR oscillation at the respiratory frequency. $b$, the power spectrum of the HR waveform which was shown in $a$. The power is given on a logarithmic scale in arbitrary units with the maximum power set to $0 \mathrm{db}$. The peaks labeled $(a)$ and (b) correspond to the very low frequency oscillation (see $a-d$ of Fig. 1a) and the low frequency oscillation (see $e, f$ of Fig. 1a) in HR. The respiratory rate at the time of this recording was in the range $0.6-0.8 \mathrm{~Hz}$, and it is seen that there is very little power associated with HRV in this frequency range.

rate, visual inspection of respiratory records indicated that a periodic modulation of breath amplitude often occurred when infants were breathing regularly. Furthermore, the frequencies of the amplitude modulation envelopes tended to be in the LF region of the $H R V$ spectra defined earlier. Consequently, we examined the relationship between variations in the amplitude of the respiratory signal and the low frequency content of HRV.

Figure 3 presents HR and respiration data taken from one of the infants studied at age $46 \mathrm{~h}$. The HR waveform for a 51.2-s period is shown in Figure $3 a$ together with the corresponding low frequency portion of its spectrum (Fig. $3 b$ ). A clear oscillation at $0.05 \mathrm{~Hz}$ is evident. The simultaneous respiration waveform is given in Figure $3 c$; but its spectrum (Fig. $3 d$ ) shows very little power at $0.05 \mathrm{~Hz}$. If, however, the points of maximum amplitude in this respiratory record are employed to form an amplitude envelope as shown in Figure $3 e$, the corresponding spectrum (Fig. $3 f$ ) has a distinct peak at $0.05 \mathrm{~Hz}$. Thus, the interaction between HR and respiration seems to occur through variation in tidal volume rather than direct RSA at the breathing frequency.
The relationship between HRV and BA appears to be strongly frequency dependent. This can be illustrated by taking two data records from the same infant. Figure 4 presents the $H R$ and $B A$ curves, along with their spectra, for a 51.2-s period during which the respiratory rate was 36 breaths $/ \mathrm{min}(0.6 \mathrm{~Hz})$ and the $\mathrm{BA}$ spectrum indicated the modulation of tidal volume was occurring predominantly at frequencies greater than $0.15 \mathrm{~Hz}$. The HRV spectrum gives the strongest response at $0.08 \mathrm{~Hz}$, corresponding to a relatively lower peak in the BA spectrum. Several of the higher frequency peaks in the latter data are also visible in the HRV spectrum, but at reduced amplitude. In contrast to this, Figure 5 gives data from the same infant taken several minutes later. Now, although the respiratory rate is the same as before, the BA modulation occurs primarily at two rather discrete frequencies, 0.06 and $0.21 \mathrm{~Hz}$. It is seen that the HRV spectrum gives a large response at $0.06 \mathrm{~Hz}$ (approximately twice the amplitude as for Fig. 4), suggesting that there may be a natural frequency, or a frequency range, for the HR control system which is amenable to excitation.

As a final example of the correspondence which can exist between HR and BA, Figure 6 presents the cross-correlation between these variables for the infant shown in Figure 3. Viewed in the time rather than the frequency domain, it is seen that the two curves vary in nearly constant phase with each other and contain a common frequency component. Each infant yielded data records which showed common frequency peaks between the HR and BA spectra. While not every record on each infant contained such a correspondence, the behavior described here was the rule rather than the exception; i.e. when a LF peak was
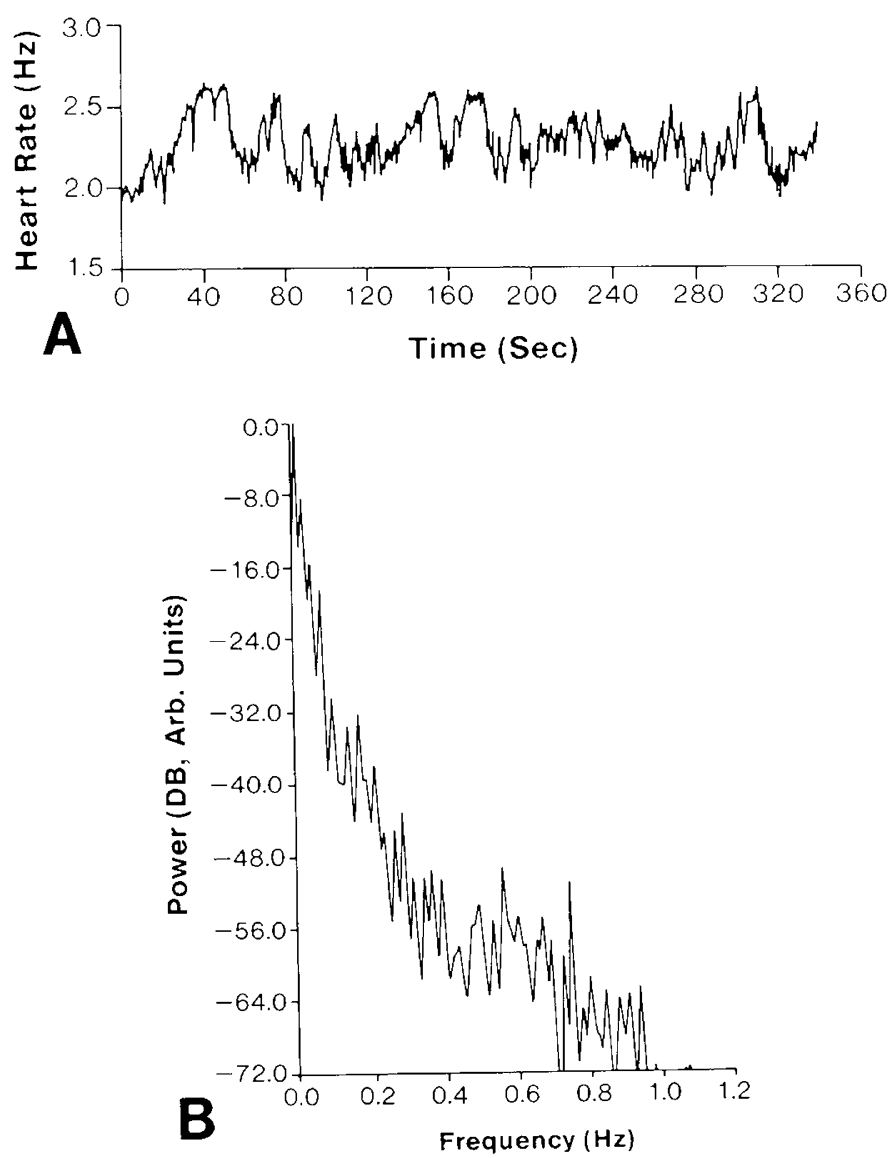

Fig. 2. a, HR of an infant 39 hours old (infant C, Table 1). Again there appear to be three frequency regions of activity. In this infant the $\mathrm{HR}$ variations at the breathing rate (RSA) can be seen more readily than for the infant of Figure $1 . b$, The power spectrum of the HR given in Figure $2 a$. Although the power of the RSA component is larger than for the infant of Figure 1, it is not a major factor in the HRV. 

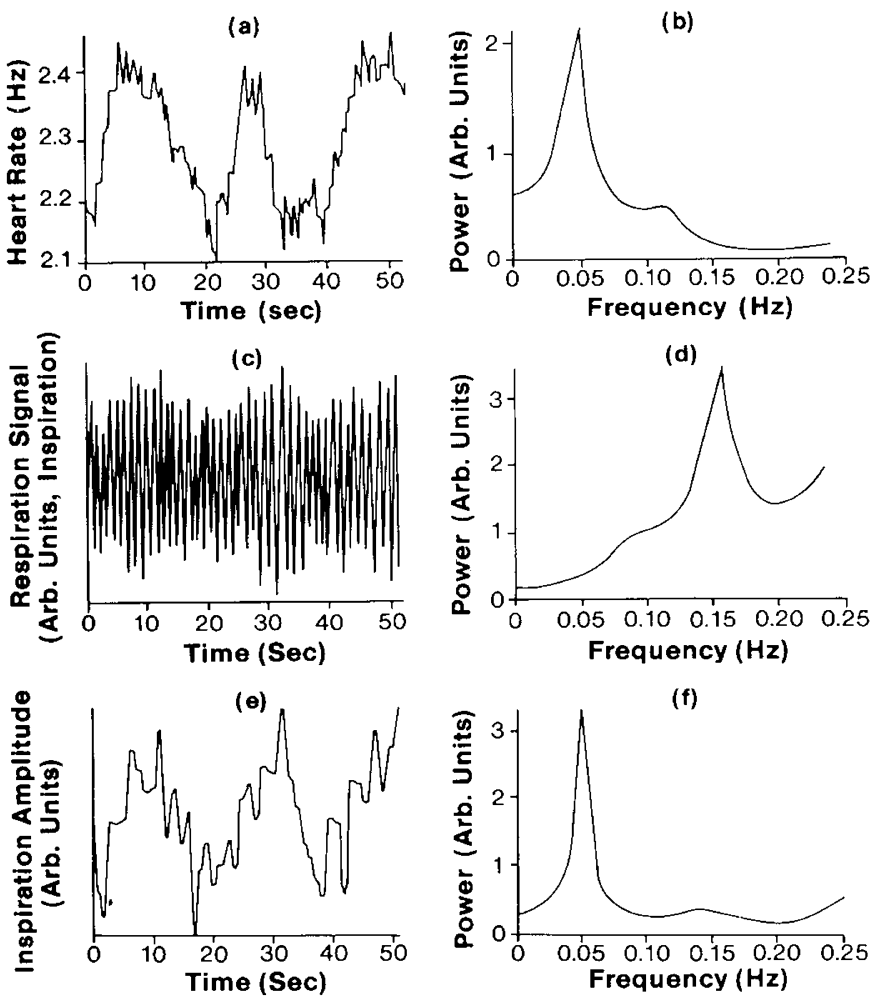

Fig. 3. Simultaneous $H R$, respiratory rate, and BA curves with power spectra from infant $\mathrm{E}$, Table 1. a shows the HR for a 51.2-s interval of recording on infant $\mathrm{E}$ at $46 \mathrm{~h}$ of age. The corresponding power spectrum (b) is given with the abscissa truncated at $0.25 \mathrm{~Hz}$ in order to illustrate better the low frequency activity. A strong peak at $0.05 \mathrm{~Hz}$ can be seen. Activity in the VLF region cannot readily be resolved with records of such relatively short length. The respiration signal, measured simultaneously with the HR, of this infant is shown in $c$. The low frequency portion of its spectrum $(d)$ indicates activity at $0.15 \mathrm{~Hz}$ but no significant power at $0.05 \mathrm{~Hz}$. (Note: if the power spectrum were presented out to, say, $1.0 \mathrm{~Hz}$, there would be a very strong component seen at the respiratory rate.) If the points of maximum amplitude (in the respiration record of Fig. 3c) are employed to form an upper envelope of the respiration record, the curve in $(e)$ results. Its power spectrum is displayed in $f$. It can be seen that a strong peak at $0.05 \mathrm{~Hz}$ occurs, corresponding to the $0.05 \mathrm{~Hz}$ in the HR spectrum of $b$.

seen in the BA spectrum, a LF peak at the same frequency usually occurred in the corresponding HR spectrum.

\section{DISCUSSION}

Although impedance measurements of respiration reflect the gas:fluid ratio in the chest, many authors have described excellent linear correlations of impedance with tidal volume at points of zero flow (end-inspiration or end-expiration) (16-19). Pulmonary blood flow increases with inspiration and provides the major intrathoracic fluid contribution to impedance while a relatively minor role is played by the intracardiac blood. However, there is minimal variation in pulmonary blood volume in the quiet, resting state (18). Since data collection in study infants occurred in quiet sleep and since data analysis of the respiration envelope selected points of zero flow (end-inspiration), the authors equated the impedance measurement of respiration with change in air volume within the chest. To confirm this decision, impedance and integrated pneumotach measurements of tidal volume were compared in a single infant. Analysis of simultaneously collected data revealed a good correlation between the two, substantiating the decision to use the impedance signal of respiration as a reflection of tidal volume in these infants.
In this study in full term neonates the HRV activity could be divided into approximately three spectral regions or frequency bands; a VLF region $(<0.02 \mathrm{~Hz})$; a $L F$ region $(0.02-0.20 \mathrm{~Hz})$; and a $\mathrm{HF}$ region $(>0.20 \mathrm{~Hz})$. The relative power in these frequency bands is tabulated for each infant in Table 1 and represents the type of HRV behavior expected during quiet sleep, but with the caveat that the behavior within any given time period may vary, depending on the specific current conditions. These data do not necessarily represent expected normal values but rather should be interpreted as values which are representative for neonates. Although there is notable variability, it is seen that approximately one-third of the HRV power is contained at frequencies below $0.02 \mathrm{~Hz}$ while nearly two-thirds appear in the frequency band from $0.02-0.2 \mathrm{~Hz}$. Of significant interest is the result that very little HRV power occurs at frequencies above 0.2 $\mathrm{Hz}$, so that there was consistently only a small degree of respiratory sinus arrhythmia.

It must be emphasized that the boundaries of these regions are precise but are based on an overall assessment of common frequency components and relative power levels. As additional knowledge is developed on specific physiological mechanisms contributing to $\mathrm{HRV}$, the various regions of activity will undoubtedly become more clearly defined. For example, in our data the HF activity, although of a low level, was invariably associated with the respiratory rate. RSA is a recognized phenomenon in healthy adults, and it is known that it decreases in magnitude as the breathing frequency increases (22). Porges (9) has suggested that RSA can be employed as a measure of vagal tone in the neurological study of infants. Kitney (11) frequently observed a significant RSA in 6-wk-old infants, and the HRV of those infants was often dominated by a large RSA occurring in the neighborhood of $0.5-0.6 \mathrm{~Hz}$. In our study of neonates less than $75 \mathrm{~h}$ old, while RSA could be identified often, it was not a large component of HRV in this early neonatal period. The largest RSA contribution measured in any of our infants was only one-fourth the amplitude (i.e. $1 / 16$ the power) of the largest low frequency component present. Our data are consistent with the findings of Haddad et al. (12) in a dog model. Although BA variations were not reported, these authors found that RRinterval power spectra in puppies showed dominant oscillations to be at low frequency and there was little RSA, whereas adult dogs displayed a significant RSA but low power at low frequencies.

On the other hand, periodic variations in the BA of our infants often correlated with dominant peaks in the LF region of the HRV spectra, suggesting that the major interaction of HR and respiration at these ages may be through a BASA, rather than a respiratory sinus arrhythmia. Nugent and Finley (10) have shown that the frequency of periodic breathing, an extreme form of breath amplitude modulation, can be detected in HRV spectra of infants and that the associated frequencies fall into the LF region. Hathorn (21) has reported fluctuations at 0.04 and 0.10 $\mathrm{Hz}$ in the tidal volume and respiratory rate of neonates. Waggener et al. (22) monitored respiration in full term and preterm infants and calculated tidal volume, breath duration, and ventilation on a breath-by-breath basis. Two types of breathing patterns were reported: type I-patterns of $18-36$ s duration $(0.056-$ $0.028 \mathrm{~Hz}$ ) associated with rhythmic changes in breath amplitude; and type II-patterns of $44-87 \mathrm{~s}$ duration $(0.023-0.011 \mathrm{~Hz})$ associated with rhythmic changes in breath duration. These investigators postulated that type I patterns represent a control phenomenon mediated by peripheral chemoreceptors while type II patterns represent respiratory control mediated by medullary chemoreceptors. Although we did not study variations in breath duration in our infants, we found that $\mathrm{BA}$ modulations tended to have their lowest prominent component in the LF range from $0.05-0.08 \mathrm{~Hz}$, a frequency band consistent with peripheral control mechanisms. In addition, our neonates frequently displayed strong VLF peaks in their HRV spectra, a frequency band compatible with chemoregulation through medullary receptors. 


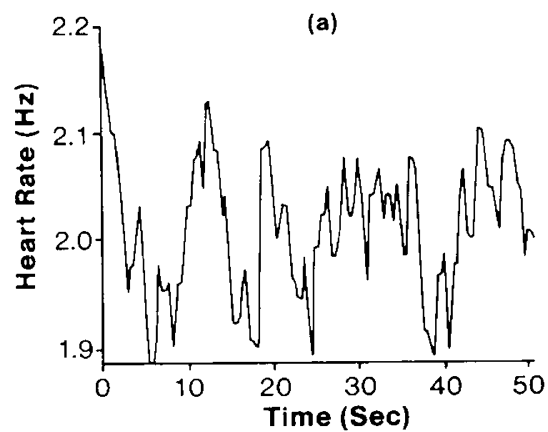

(b)
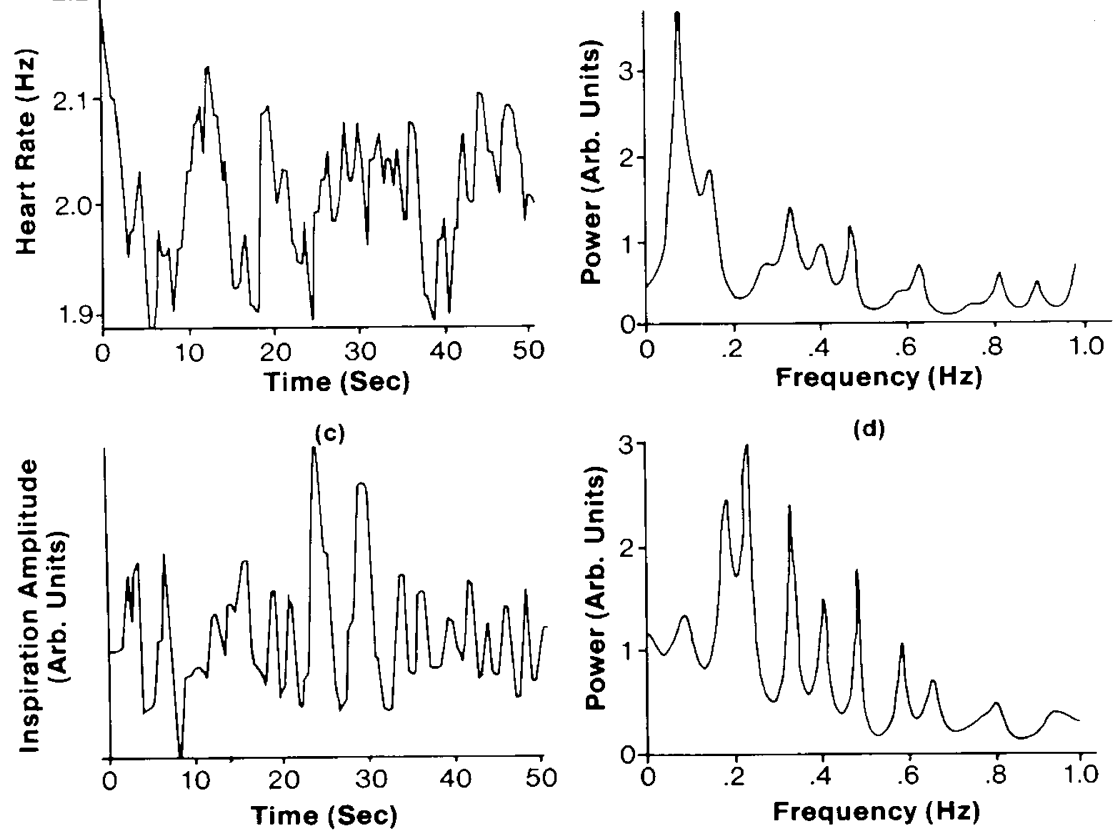

(d)

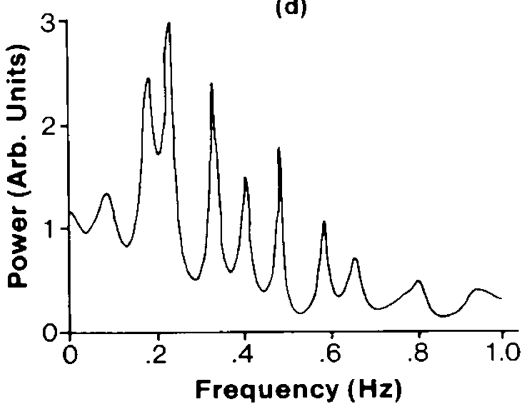

Fig. 4. Simultaneous HR and BA curves with power spectra from infant B, Table 1. The HR waveform for a $51.2-\mathrm{s}$ interval in infant B (age 39 h) is shown in $a$, and its spectrum is given in $b$. There is a strong component of HRV at $0.08 \mathrm{~Hz}$. The BA during this time is shown in $c$, and its power spectrum is presented in $d$. There is only relatively small power at $0.08 \mathrm{~Hz}$ in the BA, and stronger components occur at higher frequencies. However, the corresponding higher frequencies in the HR spectrum $(b)$ are attenuated in comparison to the $0.08 \mathrm{~Hz}$ component. This may imply that the HR control mechanism acts as a low pass filter to suppress HRV at higher frequencies.

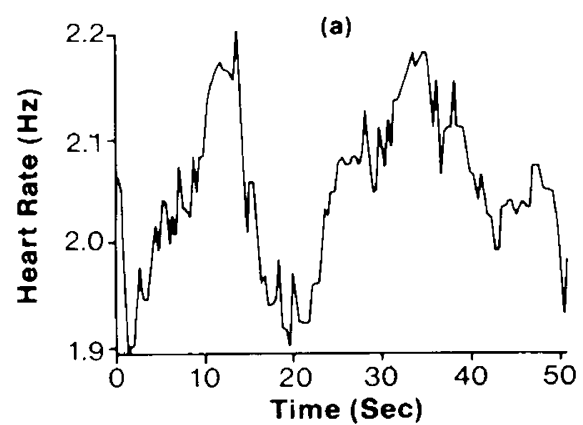

(c)

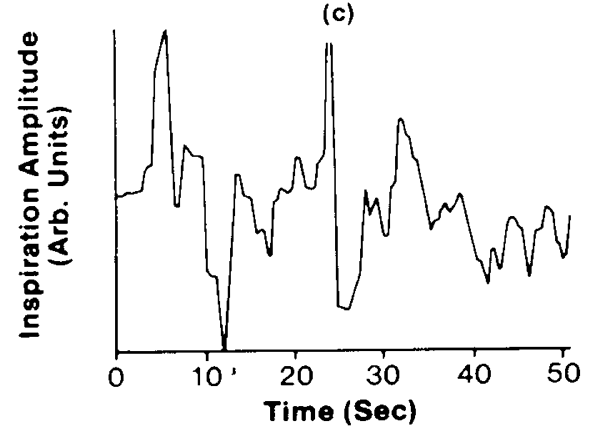

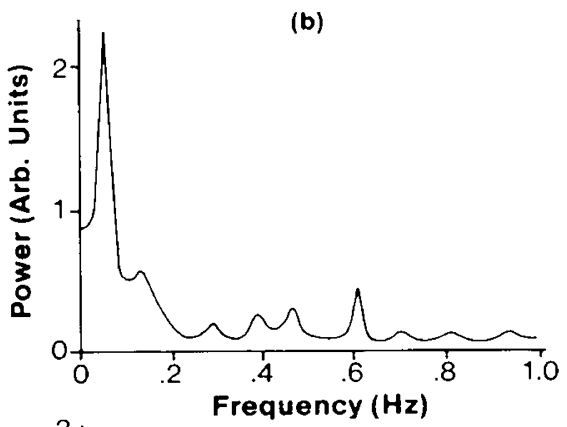

(d)

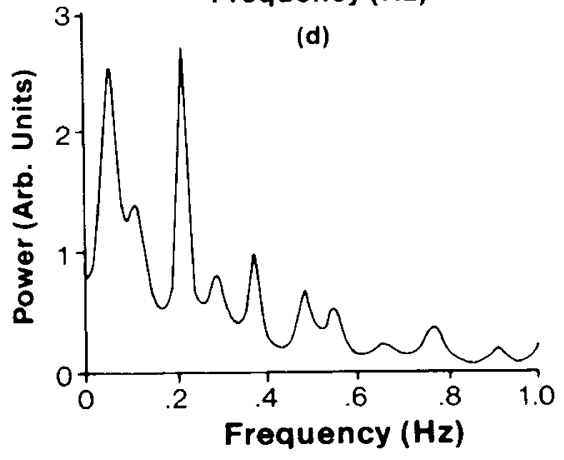

Fig. 5. Simultaneous HR and BA curves with power spectra from infant B, Table 1. This figure presents results obtained a short time later in the same infant from which Figure 4 was constructed. During this measurement interval the $\operatorname{HR}(a)$ and its spectrum $(b)$ demonstrate strong activity at $0.06 \mathrm{~Hz}$. The BA $(c)$ has large power components at 0.06 and $0.21 \mathrm{~Hz}$ as shown in $d$. Note that the $0.06 \mathrm{~Hz}$ activity is large in both $\mathrm{HR}$ and $\mathrm{BA}$, but there is little HRV at $0.21 \mathrm{~Hz}$. A small RSA at $0.6 \mathrm{~Hz}$ is seen in the spectrum of the HR.

The present study demonstrates the existence of a strong relationship between $H R$ and repiration in the first 3 days of life, not on a breath-by-breath basis, but rather with changes in the BA. When the points of peak inspiration of individual breaths were formed into a time series, there often existed a strong correlation between this curve and the HR curve. While common frequencies were evident in the lower frequency region of these
BA and HR curves when viewed in the spectral domain, the relationship between them could often best be viewed in the time records and CCFs. These records indicate the existence of three types of interactions. First, there were numerous records which showed stable oscillations at a discrete frequency and for a lengthy time in both the HR and BA signals, as illustrated by Figures $3 a-b, 3 e-f$. Second, records were observed in which both 

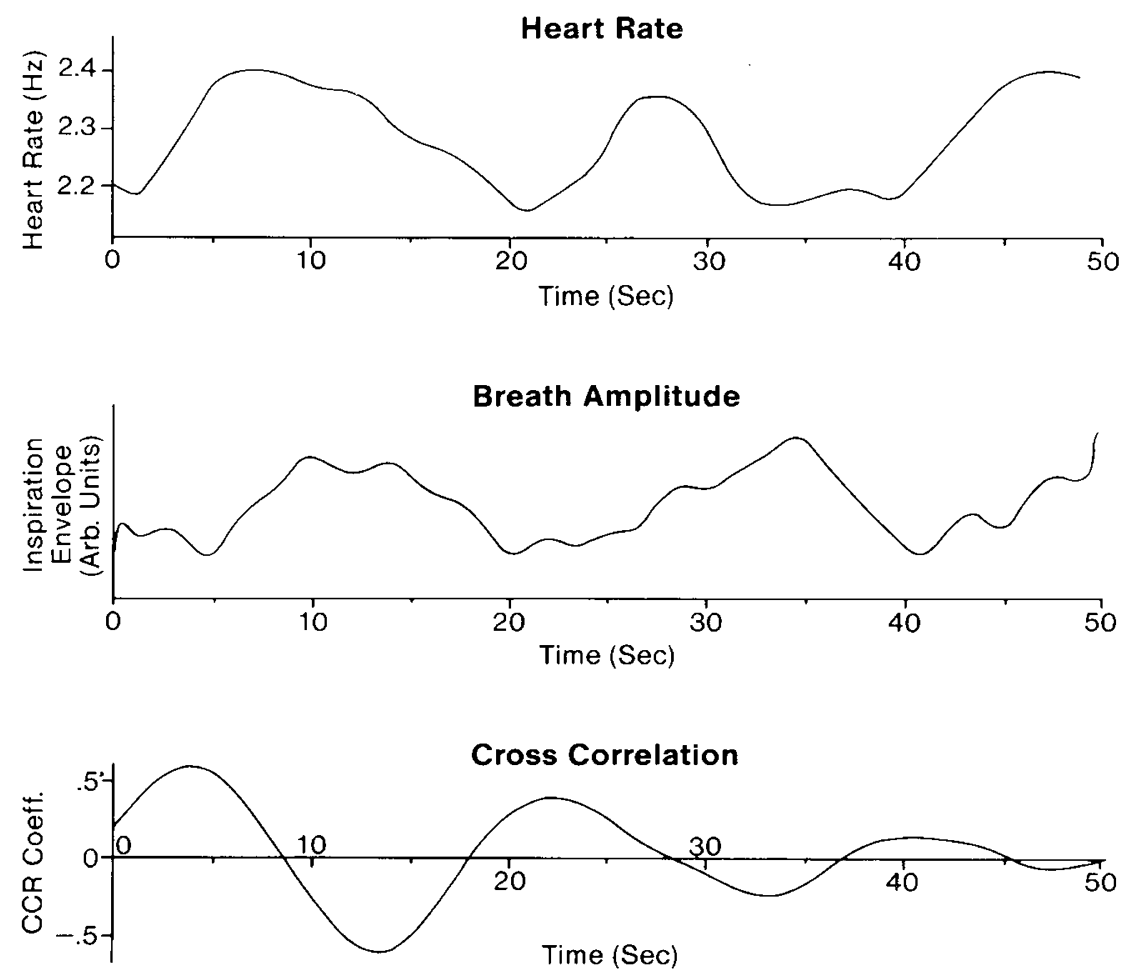

Fig. 6. HR, BA, and CCF from infant E, Table 1. The HR $(a), \mathrm{BA}(b)$ and their $\mathrm{CCF}(c)$ for the infant whose data were presented in Figure 3 . The HR and BA curves have been low pass filtered at $0.25 \mathrm{~Hz}$ to remove higher frequency variations. It is seen that the changes in BA follow those in HR by approximately $4 \mathrm{~s}$.

$\mathrm{HR}$ and BA oscillated together and at the same frequency, but for which this frequency tended to change slowly with time. Figures 4 and 5 are examples of this type of quasistable oscillation. Finally, there were curves for which neither HR nor BA tended to oscillate at clearly identifiable frequencies. These can be termed transitional in nature, since each infant exhibited behavior of a stable or quasistable nature during much of the recording.

Thus, the data from the newborn infants in this study indicate that a component of $\mathrm{HRV}$ is associated with BA variability and that HRV is not strongly influenced by the respiratory sinus arrhythmia seen in adults and older infants. One hypothesis for this behavior is that the HR control system in term neonates has a frequency range of greatest sensitivity in the neighborhood of $0.06-0.08 \mathrm{~Hz}$ within which respiratory effects, such as tidal volume modulation, breath duration or direct RSA, affect HR variability. Outside this range there is diminishing interaction as the frequency increases.

BASA may occur by two different physiological pathways which are consistent with our data and with other reported results: (i) direct action of the breath amplitude upon the heart rate (cause-effect relationship); (ii) simultaneous and similar control of heart rate and respiration by a higher order mechanism (associative relationship).

Since RSA in adults is established as a cause-effect relationship in that respiration may be viewed as a stimulus which produces an RSA component in the HR, it is possible to interpret the phenomenon of BASA in neonates in a similar fashion, i.e. to view variations in $B A$ as a stimulus or input to $H R$ control which produces an output that contains HR oscillations at corresponding frequencies. For example, the delay in the baroreceptor loop in response to a stimulus arises from neural conduction time and the response time of effector organs. It has been shown that this delay leads to a self-oscillation in the blood pressure control system at a frequency of $\sim 0.1 \mathrm{~Hz}$ in healthy adults $(7,8)$, and this oscillation is also seen in the heart rate. If a stimulus (e.g. respiration) occurs at a frequency near this baroreceptor frequency, the $0.1 \mathrm{~Hz}$ oscillation will be entrained at the respiration rate and HRV tends to be enhanced. If the stimulus is applied at a frequency which is high relative to the self-oscillation frequency, however, the control feedback has insufficient time to be sensed before the stimulus has changed and, consequently, entrainment does not occur. This is analogous to resonance in a mechanical or electrical system. The system thus acts as a filter upon the applied stimulus such that the further removed from the "natural" frequency, the less the effect from a given stimulus amplitude $(7,17)$. In neonates the respiratory rate is so high (the average respiration rate in this study was 48 breaths/min) that significant RSA may not occur due to the low pass filtering effect of the HR control system. However, stimuli at lower frequencies, such as BA and breath duration oscillations, may produce corresponding $\mathrm{HR}$ oscillations. This low pass filtering effect probably arises from immaturity of the parasympathetic nervous system and the dominance of the sympathetic nervous system in neonates (14). Thus, two factors could lead to enhanced RSA with increasing postnatal age: (i) continued maturation of the parasympathetic nervous system and (ii) decreasing respiratory rate.

An alternative possibility is that the BASA represents simply an associative relationship between breathing and $\mathrm{HR}$ in that each is being regulated simultaneously and in a similar fashion by a higher control center. For example, the data of Waggener et al. (22) support the concept that tidal volume is mediated by peripheral chemoreceptors in a frequency range from $0.028-0.56$ $\mathrm{Hz}$. Peripheral chemoregulation at these frequencies may also be responsible for HRV through the sympathetic nervous system without employing respiration as an intermediate (stimulus) pathway.

In summary, the present studies have demonstrated that: (i) HRV spectra in neonates can be divided into VLF, LF and HF regions of activity; (ii) newborn infants usually exhibit a strong VLF component of HRV; (iii) RSA, which exists in the HF 
region, is not a major factor in $\mathrm{HRV}$ in term infants during the first few days of life; (iv) HR and respiration in these infants are related through a BASA which occurs in the LF region of the spectrum.

These term newborn infants demonstrate little effect of the respiratory rate on HRV. By 6 wk of age, the spectra have changed and RSA is present and more significant. This suggests a developmental/evolving process of determinants of HRV. BA modulation influences HRV in the early neonatal period and is eventually replaced by "adult type" respiratory rate modulation. BA modulation of HR may arise either as a response of the HR control system to breath amplitude stimuli or as an association with similar but independent control of $\mathrm{HR}$ and respiration by a higher control mechanism.

Our data are consistent with the hypothesis that the newborn infant has an immature parasympathetic nervous system and an active, dominant sympathetic nervous system. We hypothesize that the strong activity in the VLF of HRV region in the term newborn is a consequence of autonomic mediation of chemoregulation, probably through medullary control. As the autonomic nervous system matures, the determinants of HRV change and the spectrum shifts to reflect this.

Thus, HRV analysis may provide a noninvasive means to study the sequential maturation of a central control mechanism during transition from periodic to controlled breathing patterns. Periodic breathing and apnea of prematurity can be considered to be extreme forms of BA modulation and may be normal variants of premature infant behavior. Conversely, sudden infant death syndrome may be the result of a failure in maturation of the relationship between HR and respiration and may be reflected in the spectra of HRV $(11,14)$. Further study of HRV in premature newborns and other infants should help us to address these speculations.

However, it cannot be overemphasized that variability in spectral content is a formidable problem to be solved before clinical utility of HRV analysis is achieved. A major impression to be gleaned from these studies as that the "normal" state of cardiorespiratory control is indeed dynamic. Not only can the distribution of HRV power vary among individuals and, within an individual, among various sleep states, but it can vary in a given individual while in a given sleep state. For example, a 250 $\mathrm{s}$ window passed through a long data record taken in quiet sleep in a particular infant might yield quite different values of the power distribution in VLF, LF, and HF bands. This observation is entirely consistent with the data of Waggener et al. (22). Therefore, great caution should be employed before accepting any particular quantitative measure of $\mathrm{HRV}$ as a reliable indicator of either normal or pathological conditions. Indeed, further research and improved basic understanding are mandatory before reliable clinical applications are forthcoming.

Acknowledgments. The authors thank Janice Ray, R.N. and Mary Elise Vigil, B.S.N., R.N. for their valuable assistance in recording and reviewing the data and Chris Sapp for her patience and diligence in preparing this manuscript.

\section{REFERENCES}

1. Bainbridge FA 1920 The relation between respiration and the pulse rate. $J$ Physiol (Lond) 54:192-202

2. Womack BF 1971 The analysis of respiratory sinus arrhythmia using spectral analysis and digital filtering. IEEE Trans Biomed Eng 18:399-409

3. Sayers BMcA 1973 Analysis of heArt rate variability. Ergonomics 16:17-32

4. Kitney RI, Rompelman O 1980 The Study of Heart Rate Variability. Oxford University Press, Oxford

5. Akselrod S, Gordon D, Ubel FA, Shannon DC, Barger AC, Cohen RJ 1981 Power spectrum analysis of heart rate fluctuation: a quantitative probe of beat-by-beat cardiovascular control. Science 213:220-222

6. Maulik D, Saini V, Zigrossi ST 1983 Clinical significance of short-term variability computed from heart-rate waveforms, J Perinat Med 11:243-247

7. Kitney RI, Linkens DA, Selman AC, McDonald AH 1982 The interaction between heart rate and respiration. Part 2 nonlinear analysis based on computer modelling. Automedica 4:141-153
8. Morguet A, Springer HJ 1981 Microcomputer-based beat-to-beat intervals and analysis of heart rate variability. Med Prog Technol 8:77-82

9. Porges SW 1983 Heart rate patterns in neonates: A potential diagnostic window to the brain. In: Field T, Sastek A (eds) Infants Born at Risk. Grune and Stratton, New York, pp 3-22

10. Nugent ST, Finley JP 1983 Spectral analysis of periodic and normal breathing in infants. IEEE Trans Biomed Eng 30:672-675

11. Kitney RI 1984 New findings in the analysis of heart rate variability in infants Automedica 5:289-310

12. Haddad GG, Jenz HJ, Lee SH, Lai TL 1984 Rhythmic variations in R-R interval during sleep and wakefulness in puppies and dogs. Am $\mathrm{J}$ Physiol 247:467-473

13. Assali NS, Brinkman CR III, Woods JR, Dandavino A 1978 Ontogeny of the autonomic control of cardiovascular functions in the sheep. In: Longo L (ed) Fetal and Newborn Cardiovascular Physiology. Garland Press, New York, pp 47-91

14. Gordon D, Cohen RJ, Kelly D, Akselrod S, Shannon DC 1984 Sudden infant death syndrome: Abnormalities in short term fluctuations in heart rate and respiratory activity. Pediat Res 18:921-926

15. Giddens DP, Kitney RI 1985 Neonatal heart rate variability and its relation to respiration. J Theor Biol 113:759-780

16. Geddes LA, Hoff HE, Hickman DM, Moore AG 1962 The impedance pneumograph. Aerospace Med 33:28-33

17. Baker LE, Hill DW 1969 The use of electrical impedance techniques for the monitoring of respiratory patterns during anaesthesia. Br J Anaesth 41:2-17

18. Victorin L, Daily W, Olsson T 1970 Transthoracic impedance: III. Methodological studies in newborn infants. Acta Paediatr Scand [Suppl] 207:37-47

19. Sandberg K, Krueger E, Lindstrom D, Sundell H, Cotton R 1985 Transthoracic impedance as a method of measuring tidal volume during high frequency ventilation. Pediatr Res 19:1826(abstr)

20. Hirsch JA, Bishop B 1981 Respiratory sinus arrhythmia in humans: How breathing modulates the heart rate. Am J Physiol 241 (Heart Circ Physiol 10):4620-4629

21. Hathorn MKS 1978 Analysis of periodic changes in ventilation in new-born infants. J Physiol 285:85-99

22. Waggener TB, Stark AR, Cohlan BA, Frantz ID, IIl 1984 Apnea duration is related to ventilatory oscillation characteristics newborn infants. $J$ Appl Physiol 57:536-544

\section{APPENDIX}

Methods of data treatment. HR and respiration data were derived from ECG and impedance measurements obtained with a Hewlett-Packard 78201B neonatal monitor. The impedance measurements reflect variations in volume of gases and fluids within the field of the ECG electrodes and as such are related to breathing amplitude. The analog signals from the monitor were recorded in a Racal Store 4 DS four channel FM recorder at 15/ $16 \mathrm{in} / \mathrm{s}$ using an IRIG wideband 1 setting. The frequency bandwith is essentially flat $( \pm 1 / 2 \mathrm{~dB})$ from $D C$ to $625 \mathrm{~Hz}$ with a 45 $\mathrm{dB}$ signal to noise ratio.

Before any digitization was performed, the analog ECG and respiration signals were played back on the RACAL instrument and recorded on a strip chart recorder for visual inspection of data quality. Sections of data with signal saturation, notable noise, or movement artifact were noted, along with the corresponding FM tape footage, and these sections were eliminated from further consideration. Digitalization of the remaining recorded signals was performed on a Cromemco microcomputer. The ECG signal was low pass filtered at $300 \mathrm{~Hz}$ with a Krohnhite Model 3343 filter at $48 \mathrm{db} /$ octave and then sampled by the microcomputer each $30 \mu \mathrm{s}$ at 12-bit accuracy. These sampled ECG values are not stored, however. Rather, an algorithm detects the local maximum value of the sampled signal, subject to the requirement that this value exceed a prescribed threshold value which is preselected after the initial review of each data record. The time of occurrence of each local maximum is determined from the internal computer clock, and this clock value is noted within the microcomputer. The process is then repeated. The next peak is detected, and the computer calculates the length of time between the occurrence of successive local maxima in the ECG signal. It is this value which is stored, and it represents the time between successive $\mathrm{R}$-waves, provided the peak detector has correctly identified the peak of the QRS complex. In most of the records analyzed, false detection-such as identifying a movement artifact as an R-wave-or missing the detection of an Rwave is not a problem due to the initial screening of data records 
for acceptable quality. However, there is no danger of either false detection or failure to detect a beat occurring unnoticed. An undetected R-wave will give rise to a sudden decrease in the heart rate curve; i.e. if one R-wave is missed, the HR drops to one-half the value of its neighboring points and this is obvious in the HR record. The procedure is then simply an ad hoc intervention by the analyst to correct an isolated missing Rwave; that is, the HR at the artifact point is reset to the average of its two adjacent values, and the time axis is shifted accordingly. If a segment of data contains several missing R-waves, the threshold level is reset to a lower value and the data are again digitized. Usually, this solves the problem. If not, that portion of the data record is discarded.

Similarly, a peak detection which does not correspond to an $\mathrm{R}$-wave is easily recognized as a sudden increase in HR. Again, for an isolated false peak this is similarly corrected. If a data record contains several successive false peaks, such as might occur with movement artifact or stretches of noisy data, that segment is eliminated from analysis. In practice there is no problem with mistakenly processing spurious peaks or missing $\mathrm{R}$-waves. The data are examined at several stages during processing, and it is easy to identify these difficulties and correct or avoid them.

What about the case where an infant might actually have an isolated arrhythmia or might skip a heartbeat? These would show up as a sudden increase or decrease in HR, respectively. If this is an isolated event, the HR value in question is set equal to the average of the adjacent $\mathrm{HR}$ value; i.e. the atypical nature of the beat is ignored. If several successive beats or arrhythmia occur often, those data are not analyzed.

The values for time between $\mathrm{R}$-waves are stored in the microcomputer and transmitted to the CYBER mainframe computer at the Georgia Institute of Technology for further processing. The reciprocal of the R-R interval gives the "instantaneous" $H R$. This results in a digital time series representation for HR but, due to variations in the rate, the time between successive HR values is not a constant. In order to use the convenience of methods applicable to equally spaced time series, the HR was linearly interpolated to equal time intervals. Womack (A1) has reported satisfactory results using this technique. Higher degree polynomial interpolations, up to fifth order, were also examined in our study, and no improvement over linear interpolation was observed. Since the HR is inherently a discretely sampled variable, the maximum frequency it is possible to measure is one-half the mean HR due to the Nyquist sampling limitation. For example, if the mean $\mathrm{HR}$ is $2 \mathrm{~Hz}$ (cycles/s) the maximum meaningful frequency in the HR power spectrum is $1 \mathrm{~Hz}$. The mean $\mathrm{HR}$ is neonates usually falls between 2 and $3 \mathrm{~Hz}$.

The question of trend detection in the data is much more subtle and much more subject to arbitrariness in setting quantitative criteria. For example, how can a trend in the data be distinguished from an oscillation frequency which is so low that only a fraction of a cycle occurs within a data record? Our procedure for selecting data records with negligible trend was accomplished in two stages. First, it was often possible to use visual inspection to discard records in which an obvious trend existed. For records which passed this criterion a second requirement was imposed, utilizing the fast Fourier transform-calculated power spectrum. When calculating the power spectrum of a record, the mean value was first removed (the mean value being defined as the time average over the record). In principle, if the data were stationary and infinitely long in duration, the dc or zero frequency value of the power spectrum would then be zero. In practice, however, various factors can contribute to a nonzero value of dc power-and one important cause is a trend in the data. Therefore, if the dc power level in the fast Fourier transform spectrum of a record exceeded $25 \%$ of the largest value of power in the spectrum, that data record was not used to compute power in the VLF band.

In addition to screening the HR records employed in the study, criteria for selecting respiration records excluded data segments which contained sighs, pauses, apnea, or movement artifact. Of course, the corresponding ECG records were also discarded. However, there was no requirement that the breathing frequency be constant for an entire data record. In fact, such records were indeed rare, although respiration records for which the breathing frequency varied by no more than $\pm 20 \%$ were common.

The respiration signal was digitized directly at 10 samples/s with 8-bit accuracy. Because this was an uncalibrated signal with regard to amplitude, the units of measure are arbitrary. The resulting digital $\mathrm{HR}$ and respiration data were recorded on a floppy disk and then transmitted to the CYBER mainframe computer at the Georgia Tech Computer Center. Further data processing was performed with the CYBER.

Two different methods of spectral analysis were employed. Because of the dynamic nature of cardiorespiratory control, the HR and respiration records are often nonstationary in nature. To demand extremely long records of stationary measurement would require eliminating large quantities of data. Also, the nonstationary behavior is frequently of interest in understanding control dynamics. Therefore, the data were treated from either of two viewpoints: (i) time or frequency domain similarities between HR and respiratory patterns and (ii) HR power distribution among various frequency bands under reasonably stationary conditions. In addressing viewpoint (i) we employed an autoregressive spectral estimator (A2, A3) and accepted data records as short as $51.2 \mathrm{~s}$, depending on the desired $\mathrm{LF}$ resolution. On the other hand, in searching for VLF oscillations, data records as long as $1000 \mathrm{~s}$ were examined. Autoregressive methods provide better identification of discrete frequency oscillations for relatively short data records than do Fourier methods. Additional details of the use of autoregressive techniques in heart rate analysis are discussed in References A4, and A5. In addressing viewpoint (ii) we employed Fourier methods of spectral estimation. This was done because autoregressive estimators were designed to perform better as frequency rather than amplitude estimators. Therefore, when reporting the percent power in various frequency bands, data records of 250 -s duration were selected which were free of significant trends according to the criteria previously described, and fast Fourier transform methods were employed to compute spectra.

No windows were convolved with the time series data for the following reasons.

1. Application of windows modifies the data, and the specific modification is dependent upon the window selected (A-6).

2. For situations in which the data were examined for oscillations of discrete frequency, a maximum entropy autoregressive method was employed. This method assumes no a priori knowledge of the data behavior outside the observation window, in contrast to Fourier methods, and it is therefore inappropriate to employ windows to reduce edge effects.

3. For situations where the percent power within various frequency bands was estimated (e.g. Table 1 of the text) the records selected were free of significant trends and of significant discontinuities at the edges. Under these conditions no major effects will result from unwindowed data, although major variations in power distribution will occur depending on the nature of the particular window used.

\section{REFERENCES}

A1. Womack BF 1971 The analysis of respiratory sinus arrythmia using spectral analysis and digital filtering. IEEE Transact Biomed Eng 18:399-409

A2. Burg JP 1972 The relation between maximum entropy spectra and maximum likelihood spectra. Geophysics 37:375-376

A3. Kay SM, Marple SL 1981 Spectral analysis-a modern perspective. Proc IEEE 69:1380-1419

A4. Giddens DP, Kitney RI 1985 Neonatal heart rate variability and its relation to respiration. $\mathrm{J}$ Theor Biol 1 13:759-780

A5. Cerutti S, Baselli G, Liberati D 1984 Autoregressive filtering in heart rate variability signal. In: Cappelini V, Constandtinides AG (eds) Digital Science Processing-84. Elsevier Science Publishers, North Holland, pp 679-683

A6. Harris FJ 1978 On the use of windows for harmonic analysis with discrete Fourier transforms. Proc IEEE 66:51-83 